\title{
Child Support, Pensions and Endogenous (and Heterogeneous) Fertility
}

\author{
András Simonovits ${ }^{1,2}$ \\ ${ }^{1}$ Institute of Economics, Centre for Economic and Regional Studies, Hungarian Academy of Sciences, Budapest, Budaörsi út 45, Hungary \\ ${ }^{2}$ Mathematical Institute, Budapest University of Technology, Budapest, Budaörsi út 45, Hungary
}

Copyright (C) 2017 by authors, all rights reserved. Authors agree that this article remains permanently open access under the terms of the Creative Commons Attribution License 4.0 International License

\begin{abstract}
In van Groezen, Leers and Meijdam (2003) (for short, GLM), the government pays child support and pensions to raise fertility and replace the insufficient old-age saving of myopic workers, respectively. Concentrating on the equilibrium core of GLM, we analyze its simplest possible versions. We impose credit constraint on workers, and extend GLM's analysis to heterogeneous rearing costs and preferences for enjoying children. Two major results: (i) the infusion of public transfers only raises social welfare when the bulk of private savings has been crowded out; (ii) the introduction of fertility-dependent pensions raises average fertility but diminishes welfare.
\end{abstract}

Keywords Child Support, Endogenous Fertility, Overlapping Generations, Pensions, Heterogeneity of Fertility

JEL Numbers D10, H55, J13, J14, J18, J26

\section{Introduction}

The worldwide population aging is a great challenge to the pension systems of the 21st century (cf. Barr and Diamond, 2008). One cause of aging is the drop in the total fertility rate. The other cause is the steep rise in life expectancy even after eliminating infant mortality. The indexation of retirement age to life expectancy is an obvious tool to tackle this second problem. But the solution of the first problem needs another tool, which enhances fertility. There are numerous empirical studies (e.g. Cigno, 1992 and Boldrin, De Nardi and Jones (2005)) claiming that the more generous the pension system (equivalently, the higher the replacement rate, i.e. the ratio of the pension to the net wage), the lower is the endogenous fertility. This gives rise to the policy proposal to introduce child allowances (more generally, support) and especially fertility-dependent pensions. It is difficult to verify these claims because the concept of child support includes a lot of different things: proper family allowances, government financed schools, free child health care etc. There may also be moral objections to excessive government intervention into family issues.

The present paper formulates a family of simplest (though not simple) models to help understanding the impact of such proposals. The basic insights are as follows: (i) too little positive transfer may be worse than no transfer at all and (ii) the fertility-dependent pensions may excessively increase the heterogeneity of fertility, while diminishing social welfare.

A lot of theoretical papers have analyzed the problem of endogenous fertility. Some of them (starting with Leibenstein (1957) and Becker (1960)) simply put the number of children into the utility function without studying the issue of pensions (cf. Eckstein and Wolpin, 1985). In contrast, van Groezen, Leers and Meijdam (2003) (for short, GLM) set up an overlapping generations model, where in each period the government collects labor taxes and pension contributions (called PAYG-taxes by GLM) from workers and in turn pays child allowances to workers and pension benefits to pensioners; the two transfers are the Siamese twins of the transfer system. (It is noteworthy that Boldrin et al. (2005, p. 0) assumed that "parents procreate because children care about his/her parents' utility, and thus provide them with oldage transfers.")

To simplify calculations, the bulk of the literature assumed that the father/mother rear his/her sons/daughters in a onesex "family". Assuming away productivity growth and identifying the lengths of old age and of young (working) age, the pension benefit is equal to the product of the contribution and the number of children. Identifying the lengths of raising children and of young age, the tax is equal to the product of the per-child family allowance and of the fertility. Note that we have assumed that these two transfer systems are balanced separately.

Furthermore, retirees decumulate their private savings to add to their benefits. Considering a small open economy, the interest rate is given and it is frequently assumed that pri- 
vate savings are more efficient than public pensions. When choosing their fertility and private saving, workers maximize their discounted lifetime utility functions, consisting of logarithmic utilities derived from young- and old-age consumption and having children - taking the support and benefits as given. In the spirit of Stackelberg's dynamic game, at the first stage, the government (leader) chooses the transfers, and at the second stage, the workers (followers) make their private decisions. But the government anticipates the workers' choices, and selects the transfers to maximize the expected social welfare. In an open economy, external debt should be considered; while in a closed economy (studied e.g. by van Groezen and Meijdam, 2008), the interest rate is endogenous.

The above-mentioned equilibrium model was only a building block of GLM (Section 3), and GLM's main emphasis was how to generate long-term social optimum. They determined the first-best optimum and tried to achieve it via markets by introducing an appropriate child support and pension system. Their basic proposition 1 states that depending on the value of the social discount factor, whether it is higher than, equal to or lower than its critical value, both optimal transfers are positive, zero or negative, respectively.

The framework of infinite generations is quite widespread in the literature but seems to be artificial. (A very ambitious study by Rangel (2003) applied the tools of game theory to model the interaction of forward and backward directed intergenerational transfers.) We only mention two pitfalls: (i) the key parameter of social discount factor is quite elusive (see the rich literature on global warming); (ii) the transfer systems of the 20th century suffered from permanent changes in the demographic and economic parameter values, entirely neglected by GLM!

This may led a number of followers to confine their attention to the equilibrium part. Reviewing the related literature, we shall also follow suit. Fenge and Meier (2005) and (2009) proved that the family allowances and fertility-related pensions are equivalent for earnings-related pensions but the former is inferior to the latter for flat pensions. (Note the very specific meaning of the words fertility- and earningsrelatedness in the case of homogeneous populations, where every worker has the same fertility and the same earning!) Galasso, Gatti and Profeta (2009) studied a similar model and demonstrated theoretically as well as econometrically that the lower the efficiency of the private savings, the larger is the decrease in fertility due to pension generosity.

When studying a related model with general rather than logarithmic utility function, Cremer, Gahvari, and Pestieau (2006) and (2008), the latter for short, CGP) also took into account that the government has no information on rearing cost differences. Applying the methods of mechanism design, they also analyzed the problem of moral hazard (when parents have not full control over their fertility) and adverse selection (when low-cost type pretend to be high-cost type). They found that "in the absence of moral hazard problems, the case for a positive link between pension benefits and fertility is not as strong as it may at first appear and as it has been advocated in some recent work" (CGP, p. 962.).

The present paper also focuses on the equilibrium part and also discusses four models of the GLM family, assuming Cobb-Douglas-utility function. The latter assumption is not only an adoption from GLM but greatly simplifies the calculations; at the same time, it weakens the realism of the models.

To avoid misunderstanding, notwithstanding our reference to GLM, we do not require that the reader had already read it. Confining attention to modern economies, we exclude negative child allowances and negative pensions. To make GLM's framework more realistic and to avoid the trap of no transfer is the best transfer, we introduce a credit constraint, excluding negative savings. (The only important exception is mortgages, generally neglected in the literature.) The credit constraint is called slack, when the saving intention is positive; tight, when the intention is negative; but in the second case, the actual saving is zero. Introducing a paternalistic social welfare function (á la Feldstein (1985) as modified by CGP), where discounting is eliminated, the socially optimal contribution and tax rates can also be determined explicitly.

In model 1 , as a rule, the optimal fertility is an increasing function of the tax rate and a decreasing function of the contribution rate. Turning to the welfare maximization, there are two local maxima: one in the slack region, namely the no-transfer case; the other in the tight region, where the two optimal transfer rates are also equal! (Compare the latter to GLM's proposition 1, where the optimal subsidy to parent is equal to the present value of the child's contribution to the intergenerational redistribution system.) For sufficiently low discount factors, the latter gives higher welfare than the former does. There is a surprising feature of these types of models (cf. Simonovits, 2013): increasing the transfer rates, first the social welfare decreases, then passing from the slack region to the tight region, it increases until reaching the global optimum.

Analyzing fertility-dependent transfers, it is natural to introduce heterogeneous actors. In models 2-4 we assume two types, the first has lower rearing cost and higher utility of having children than the second has. As a good approximation, we shall assume that the type-specific child allowances are proportional to the type-dependent fertilities. To analyze fertility-specific pensions, we also assume that the pension benefit is a convex linear combination of two schemes: a common and an individualized one, the latter being proportional to the type-specific fertility. To get rid of the problems of having a slack credit constraint for one type and a tight one for the other, we concentrate on the pure combinations. Moreover, we only touch the slack-slack pair and confine our attention to the simplest case of tight-tight combination. (Note, however, that if the heterogeneity is strong, and the pension is strongly proportional to the type-specific fertility, then the low-fertility type must save.) We shall see that the equilibrium fertilities satisfy our expectations: type 1 has more children than type 2 has. (Simonovits (2014) concentrates on the fertility block and demonstrates that with common preferences for children and growing heterogeneity in rearing costs, the higher cost type pays more and more net transfer to the other type.)

Model 2 generalizes model 1 to heterogeneous fertility but 
retains homogeneous pensions. Here the determination of the common per-child allowance requires further analysis. Model 3 introduces fertility-specific pensions, but neglects its incentive effects. By increasing the variance of pensions, without raising the endogenous fertilities, such a reform trivially decreases the social welfare for every pair of transfer rates. In contrast, model 4 introduces incentive effects into model 3 ending up with a game-theoretic model. In the simplest and most interesting case of total separation of the two types' pensions, however, model 4 can be reduced to model 3. It is analytically not clear whether the higher and more diverse fertility increases or not the welfare. Moreover, the social tensions arising between low- and high fertility types may prevent the introduction of such a system.

To have a better understanding of the differences among GLM and our four models, Table 1 displays their most important characteristic features. Though the lack of dynamics is generally a minus, here it is denoted as a plus, because this is the key to get rid of the problematic aggregation across generations (column 1). The presence of heterogeneity is a welcome step toward modeling redistribution via family allowances (column 2) and via fertility-dependent pensions (column 3). The incorporation of incentives distinguishes M4 from M3 (column 4).

Table 1. Characteristics features of GLM and M1-M4

\begin{tabular}{lcccc}
\hline & & & \multicolumn{3}{c}{ Differentiated pensions } \\
Model & Static & Heterogeneous & no incentives & with incentives \\
\hline GLM & - & - & - & - \\
M1 & + & - & - & - \\
M2 & + & + & - & - \\
M3 & + & + & + & - \\
M4 & + & + & + & + \\
\hline
\end{tabular}

To have a feeling for the order of magnitudes, we shall display several numerical calculations following our theoretical results. The simplifications mentioned above exclude any serious calibration. Nevertheless, our numbers will help to check if our models are sensible or not. Moreover, analytical results like this variable is a decreasing function of another variable may not be that interesting in themselves. What is really important is how strong the dependence is.

Using an ad hoc parameterization, we really get a feeling for the numbers. For example, comparing the maximal welfares in models 3 and 4 to model 2, the introduction of pensions proportional to fertilities, the efficiency drops by 6 and 2 , percents, respectively.

We call attention to several limitations of the models of this type: (a) the role of social fertility norms is totally neglected, (b) wage differences are overlooked, (c) linear, mostly proportional taxes and benefits are assumed and (d) the coexistence of one- and two-parent families are forgotten; (e) the impact of survivor's pensions is skipped over. Concerning (a), it is obvious that deciding on the number of children, the would-be parents are much more influenced by their social environment than when they decide on the type of car they buy. (For the issue of social norms in the welfare state, see Lindbeck, Nyberg and Weibull (1999).) Concerning (b), uniform family allowances redistribute from the higher-paid to the lower-paid, while tax allowances do the opposite. Concerning (c), in some countries, the family benefit and especially the tax allowance are strongly nonlinear. (For example, since 2011, in Hungary, the birth of the third child more than triples the maximal per-child tax allowance!) Concerning (d), the rearing costs are basically different in a one- and a two-parent family. Concerning (e), the existence of survivor's pensions is a significant pro-family tool and there are proposals to diminish or eliminate it.

The structure of the remaining part of the paper is as follows. Sections 2 discusses homogeneous fertility. Section 3 studies heterogeneous endogenous fertility. Section 4 concludes. An Appendix contains the more technical proofs.

\section{Homogeneous Fertility}

In this section we recapitulate the equilibrium part of GLM based on the usual two-period overlapping generations model with homogeneous fertility, modify and develop it further.

\subsection{Framework}

We keep GLM's notations except for replacing their young and old-age consumption $c^{y}$ and $c^{o}$ by the simpler $c$ and $e$, respectively and abbreviating their interest factor $1+r$ by $R$. It is heroically assumed that the tax and contribution rates do not influence the labor supply which is equal to unity. Furthermore, in our small open economy, the world interest rate determines the domestic interest rate and together with the other variables, the steady state per worker capital and external debt.

Total wage: $w=1$, pension contribution (rate): $\tau$, tax (rate): $\theta$, saving: $s$. The cost of rearing a child: $p$, per child benefit: $\varphi$, fertility, i.e. the number of children per parent: $n$; the pay-as-you-go benefit: $\eta$; all positive real numbers. Another heroic assumption: the number of children can be any positive real!

Young-age $(c)$ and old-age consumptions $(e)$ are respectively

$$
c+(p-\varphi) n=1-\tau-\theta-s \text { and } e=R s+\eta .
$$

Note that in a related model without old-age, Simonovits (2015) improved the raising function, making it proportional to the net income with proportionality factor $\pi>0$ :

$$
c=(1-\pi n)(1-\tau-\theta+\varphi n), \text { where } 0<n<\frac{1}{\pi} .
$$

The lifetime utility function is additively logarithmic:

$$
U(c, n, e)=\log c+\gamma \log n+\beta \log e,
$$

where $\gamma$ is the coefficient of the relative utility of having children and $\beta$ is the discount factor, $\gamma>0$ and $0 \leq \beta \leq 1$. 
(Note, however, that this utterly simple description does not take into account the welfare of the dependent children: their rearing cost can be low because the parents are either efficient or save on necessities.)

Introducing the net of contribution wage $\hat{\tau}=1-\tau$ and inserting (1) into (2) yield the reduced utility function:

$u(s, n)=\log (\hat{\tau}-\theta-s-(p-\varphi) n)+\gamma \log n+\beta \log (R s+\eta)$.

We assume that our small open economy operates with per capita capital $k$, production $f(k)=1$ and external debt ratio $d$. Then we have

$\left(1-n^{-1}\right) k=1-c-n^{-1} e-p n+s+(n-R) d$ with $k>0$.

Denoting the transfer-dependent individual optimum triple by $c(\tau, \theta), n(\tau, \theta), e(\tau, \theta)$ and adopting the idea of Feldstein (1985), we define a paternalistic social welfare function by replacing the discount factor $\beta$ with 1 in (2). Then our social welfare function is

$$
V(\tau, \theta)=\log c(\tau, \theta)+\gamma \log n(\tau, \theta)+\log e(\tau, \theta) .
$$

Following GLM, in our basic model, the workers neglect the impact of their decisions on the balances described in (5) below. Filling the gap left in GLM, eventually we shall exclude negative savings, and distinguish two cases: either slack or tight credit constraint.

To learn if the credit constraint is slack or tight, we must determine the separatrix curve $\theta(\tau)$ which separates the two domains in the $(\tau, \theta)$-plane.

Lemma 1. 1. a) The separatrix curve $\theta(\tau)$ is given by

$$
\theta(\tau)=\frac{\left[\beta p-\gamma R^{-1} \tau\right] \hat{\tau}}{\beta p+R^{-1} \tau}, \text { where } 0 \leq \tau<\gamma^{-1} \beta p R
$$

b) The separatrix curve starts from $\theta(0)=1$ and ends at $\theta\left(\tau_{M}\right)=0$, where $\tau_{M}=\gamma^{-1} \beta p R$; and $\theta(\tau)$ is declining in $\left[0, \tau_{M}\right]$.

c) If $0 \leq \tau<\tau_{M}$ and $0<\theta<\theta(\tau)$, then $s(\tau, \theta)>0$ : slack. tight.

d) If $0 \leq \tau \leq \tau_{M}$ and $\theta(\tau) \leq \theta \leq 1-\tau$, then $s(\tau, \theta)=0$ :

e) If $\tau_{M}<\tau \leq 1-\theta$, then $s(\tau, \theta)=0$ : tight.

The proof is given in the Appendix but only after the proofs of Theorems 1 and 3 .

\subsection{Slack credit constraint (S)}

We continue the analysis with the slack credit constraint. Copying GLM, we take the partial derivatives of $(2 *)$ with respect to $s$ and $n$ yields the first-order necessary conditions for optimum:

$$
0=u_{s}^{\prime}(s, n)=\frac{-1}{\hat{\tau}-\theta-s-(p-\varphi) n}+\frac{\beta R}{R s+\eta}
$$

and

$$
0=u_{n}^{\prime}(s, n)=\frac{-(p-\varphi)}{\hat{\tau}-\theta-s-(p-\varphi) n}+\frac{\gamma}{n} .
$$

For the time being, we do not exclude negative savings. Then the conditional optima are

$$
\begin{gathered}
c(\tau, \theta)=\frac{\hat{\tau}-\theta+R^{-1} \eta}{1+\beta+\gamma}, e(\tau, \theta)=\beta R c(\tau, \theta) \\
\text { and } n(\tau, \theta)=\frac{\gamma c(\tau, \theta)}{p-\varphi} .
\end{gathered}
$$

Here pension $\eta$ and per-child allowance $\varphi$ depend on fertility $n$, and we took them as given. Now we introduce the transfer equations mentioned in the Introduction:

$$
\theta=\varphi n \text { and } \eta=\tau n
$$

In words: a) the tax rate is equal to the product of the perchild family allowance and of the fertility; b) the pension benefit is equal to the product of the contribution and the number of children.

The introduction of per-child support $\varphi$ reduces the private cost of rearing a child from $p$ to $p-\varphi$. If (5a) holds, there is no income effect. The introduction of the contribution rate $\tau$ diminishes the young-age consumption by the same amount and increases the old-age consumption according to $\eta=\tau n$ [(5b)]. In a stationary economy with $n=1$, the two changes cancel each other; for falling/growing population, the reduction of young-age consumption is greater/lower than the increase of the old-age consumption. Using the concept of dynamic efficiency: $R>n$, the comparison above changes. As is known, in a dynamically efficient economy, the introduction of a pay-as-you-go pension is suboptimal etc.

Inserting the transfer equations (5a)-(5b) into (3a)-(3b) yields the final optima:

Theorem 1. (GLM, p. 241.) a) When the credit constraint is slack (Lemma 1c), then the individually optimal consumption pair and fertility are given respectively.

$$
\begin{gathered}
c_{S}^{*}=\frac{\hat{\tau}-\theta[1-\tau /(p R)]}{1+\beta+\gamma-\gamma \tau /(p R)}, \quad e_{S}^{*}=\beta R c_{S}^{*} \\
\text { and } n_{S}^{*}=\frac{\gamma \hat{\tau}+(1+\beta) \theta}{(1+\beta+\gamma) p-\gamma R^{-1} \tau} .
\end{gathered}
$$

b) For a given contribution rate $\tau$, the optimal fertility is an increasing function of the tax rate $\theta$. For a given tax rate $\theta$, the optimal fertility is a decreasing/increasing function of the contribution rate if the tax rate is lower/higher than the critical tax rate

$$
\theta^{\mathrm{o}}=\frac{(1+\beta+\gamma) p R-\gamma}{1+\beta}
$$

Remarks. 1. If $\theta^{\circ}<0$, then the first case (decreasing fertility: normal) is empty; if $\theta^{\circ}>\theta(\tau)$, then the second case (increasing fertility: paradox) is empty. Denoting by $\tau^{\circ}$ the root of $\theta^{\circ}=\theta(\tau)$, to be called critical contribution rate, normalcy prevails in interval $\tau^{\circ}<\tau<1$. 
2. The child support is always lower than the expenditure on children: $0 \leq \varphi<p$. (This also holds in the tight region.)

Though we are unable to give a full picture of the dependence of social welfare on the transfer rates in the slack region, we prove that the no-transfer system is a (local) maximum if $\gamma$ is not too large.

Theorem 2. If $\gamma<2$, then the no-transfer system $\left(\tau_{S}^{*}, \theta_{S}^{*}\right)=$ $(0,0)$ is a local maximum, where the optimal outcomes are

$$
\begin{gathered}
c_{S}^{* *}=\frac{1}{1+\beta+\gamma}, \quad e_{S}^{* *}=\frac{\beta R}{1+\beta+\gamma} \\
\quad \text { and } n_{S}^{* *}=\frac{\gamma}{(1+\beta+\gamma) p} .
\end{gathered}
$$

Remarks. 1. The assumption $\gamma<2$ seems to be quite mild.

2. Probably our local maximum is also a global one in the slack region (Tables 4 and 5 below).

\subsection{Tight credit constraint (T)}

We turn now to the tight credit constraint neglected by GLM: $s=0$. We must replace condition $u_{s}^{\prime}(0, n)=0[(3 \mathrm{a})]$ by $u_{s}^{\prime}(0, n)<0$ and then $(3 \mathrm{~b})$ becomes $u_{n}^{\prime}(0, n)=0$. By easy calculation we obtain

$$
(p-\varphi) n=\gamma c=\gamma[\hat{\tau}-\theta-(p-\varphi) n] .
$$

Inserting the budget condition $\theta=\varphi n$, and the notation $\bar{\gamma}=$ $1+\gamma$, we end-up with

Theorem 3. a) When the credit constraint is tight (Lemma $1 d+e)$, the optimal fertility and the corresponding consumption pair are respectively

$$
\begin{gathered}
n_{T}^{*}=\frac{\gamma \hat{\tau}+\theta}{\bar{\gamma} p}, \quad c_{T}^{*}=\frac{\hat{\tau}-\theta}{\bar{\gamma}} \\
\text { and } e_{T}^{*}=\tau n_{T}^{*} .
\end{gathered}
$$

b) The optimal fertility is a decreasing function of the contribution rate and an increasing function of the tax rate.

c) For $0<\gamma<1$, the equal increase of the transfer rates increases the optimal fertility: $\Delta \tau=\Delta \theta>0$ implies $\Delta n_{T}^{*}>0$, while for $\gamma>1$, the opposite happens: $\Delta n_{T}^{*}<0$.

Next we determine the socially optimal pair of contribution and tax rates in the tight region.

Theorem 4. In the region of tight credit constraint, the socially optimal contribution and tax rates are identical:

$$
\tau_{T}^{*}=\theta_{T}^{*}=\frac{1}{3+\gamma}
$$

where the optimal outcomes are

$$
\begin{gathered}
n_{T}^{* *}=\frac{\gamma}{(1+\gamma) p}, \quad c_{T}^{* *}=\frac{1}{3+\gamma} \\
\text { and } e_{T}^{* *}=\frac{1}{(3+\gamma)^{2} p}
\end{gathered}
$$

Remarks. 1. It is easy to show that the optimal pair of transfer rates in (9) generate tight credit constraint.

2. It is natural that the socially optimal fertility $n_{T}^{* *}$ is a decreasing function of the relative utility of children but it is a specific feature of the model that the two optimal transfer rates are equal to each other and are independent of any other parameter value.

3. In a prototype model without old age, Simonovits (2014) also introduces a paternalistic coefficient $\gamma^{*}$, to derive extra children.

Comparing the welfare maxima of the two regions, the following quantity plays an important role. Function $\beta(p, \gamma, R)$ determines the critical discount factor as the function of the rearing cost, of the relative child utility and of the interest factor, where the two optimal systems achieve the same social welfare. Function $\beta(p, \gamma, R)$ is defined by the following implicit equation (cf. $\left(6^{*}\right)$ and $\left.\left(8^{*}\right)\right)$ :

$$
V(0,0, \beta)=V\left(\tau_{T}^{*}, \theta_{T}^{*}, \beta\right)
$$

but its theoretical investigation seems to be hopeless.

Obviously, if $0<\beta<\beta(p, \gamma, R)$, then the T-optimum is better than the $\mathrm{S}$-optimum, and vice versa.

\subsection{Single Instruments and Relative Efficiency}

It may be helpful further analyzing the two very important special cases, namely when either a) there is no child-support or b) there is no pension.

a) No child-support: $\theta=0=\varphi$

By Lemma 1, the optimal saving $s>0$ if $0 \leq \tau<$ $\gamma^{-1} \beta p R$, zero otherwise. Then the optimal adult consumption and fertility of the two regions are respectively equal to

$$
c_{S}^{*}=\frac{\hat{\tau}}{1+\beta+\gamma-\gamma \tau /(p R)} \text { and } n_{S}^{*}=\frac{\gamma \hat{\tau}}{(1+\beta+\gamma) p-\gamma R^{-1} \tau}
$$

and

$$
n_{T}^{*}=\frac{\gamma \hat{\tau}}{\bar{\gamma} p} \text { and } c_{T}^{*}=\frac{\hat{\tau}}{\bar{\gamma}}, \quad \hat{\tau}=1-\tau .
$$

b) No pensions: $\tau=0$ and $\eta=0$

By Lemma $1, \theta(0)=1$, i.e. for any $0 \leq \theta \leq 1$, the optimal saving is positive and the optimal adult consumption and fertility are respectively equal to

$$
c_{S}^{*}=\frac{1-\theta}{1+\beta+\gamma} \text { and } n_{S}^{*}=\frac{\gamma+(1+\beta) \theta}{(1+\beta+\gamma) p} .
$$

Note that the extreme pair $(\tau, \theta)=(0,1)$ provide the highest fertility: $n_{S}^{*}=1 / p$.

As is well-known, the numerical value of the social welfare function has no economic content. To obtain meaningful numbers, we shall occasionally compare the welfare provided by a $(\tau, \theta)$-system with the no-transfer system's $(0,0)$ as follows. Let us define the relative efficiency of the former with respect to the latter by the positive number $\varepsilon(\tau, \theta)$ if multiplying the unit wage by $\varepsilon$ in the no-transfer system, 
the welfare would reach that value provided by the transfer system with unitary wages. In formula:

$$
V[\varepsilon, 0,0]=V[1, \tau, \theta] .
$$

Due to the simple utility function (2), the optimal fertility is independent of the wage and the optimal consumption pair are homogeneous linear functions of the wage. Therefore

$$
\begin{gathered}
2 \log \varepsilon(\tau, \theta)+\log c(0,0))+\gamma \log n(0,0)+\log e(0,0) \\
=\log c(\tau, \theta)+\gamma \log n(\tau, \theta)+\log e(\tau, \theta) .
\end{gathered}
$$

Hence $\log \varepsilon(\tau, \theta)$ or $\varepsilon(\tau, \theta)$ can simply be determined:

$$
\begin{gathered}
2 \log \varepsilon(\tau, \theta)+V[1,0,0]=V[1, \tau, \theta] \text { i.e. } \varepsilon(\tau, \theta) \\
=\exp [0.5(V[1, \tau, \theta]-V[1,0,0])]
\end{gathered}
$$

\subsection{Numerical Illustrations}

To have a feeling for the order of magnitudes, we shall display several illustrative numerical calculations. Note that our contribution and tax rates are higher than they should be, because we do not scale down the length of the periods spent with raising children (length of 20 years) and drawing pen-

\begin{tabular}{|c|c|c|c|}
\hline Coefficient of & Compound & Critical & Optimalsteady \\
\hline relative & interest & discount & state fertility \\
\hline child utility & factor & factor & slack tight \\
\hline$\gamma$ & $R$ & $\beta$ & $n_{T}^{*}$ \\
\hline \multirow[t]{3}{*}{0.3} & 1.1 & 0.300 & 0.536 \\
\hline & 1.3 & 0.229 & $0.561 \quad 1.126$ \\
\hline & 1.5 & 0.186 & 0.577 \\
\hline \multirow[t]{3}{*}{0.4} & 1.1 & 0.366 & 0.647 \\
\hline & 1.3 & 0.272 & $0.684 \quad 1.176$ \\
\hline & 1.5 & 0.218 & 0.706 \\
\hline \multirow[t]{3}{*}{0.5} & 1.1 & 0.441 & 0.736 \\
\hline & 1.3 & 0.316 & $0.787 \quad 1.224$ \\
\hline & 1.5 & 0.250 & 0.816 \\
\hline
\end{tabular}
sions (length of 20 years) with respect to the working period (length of 40 years). To give room for an unfunded pension system, we assume strong myopia: $\beta R<1$.

Table 2. Critical discount factor function $\beta(\gamma, R)$

Remark. $p=0.35$.

Table 2 displays the critical (compound) discount factor for a fixed rearing cost $p=0.35$ for selected points of a grid in the $(R, \beta)$-plane. For a fixed $\gamma$, the higher the (compound) interest factor, the lower is the critical value. For example, for $\gamma=0.4$, as the 30-year compound interest factor $R$ rises from 1.1 to 1.5 , the critical $\beta$ drops from 0.366 to 0.218 . In parallel, the steady state fertility rises from $n_{S}^{*}=0.647$ to 0.706 , while $n_{T}^{*}=1.176$ remains invariant. Similarly, fixing $R=1.3$, as $\gamma$ rises from 0.3 to 0.5 , the critical $\beta$ rises from 0.229 to 0.316 .

Example 1. Choose the middle (italicized) row in Table 2: $\gamma=0.4, R=1.3$ (the annual interest rate being $0.88 \%$ ), and $\beta=0.2$ (i.e. the annual discount rate of $5.2 \%$ ), well below the critical value 0.272 (annual discount rate 4.3\%).

Varying the contribution and the tax rates, their impact can be studied also numerically. First we present the separatrix (discussed in Lemma 1) which separates the $\mathrm{S}$ and $\mathrm{T}$ regions. It declines from 1 to 0 while the contribution rate $\tau$ rises from 0 to 0.23 . The critical tax rate $\theta^{\circ}=0.273$ and the corresponding contribution rate $\tau^{\mathrm{O}}=0.09$. Second, from Theorem $4, \tau^{*}=\theta^{*}=0.294$, close 0.3 , appearing in Table 4 below.

To stay close to the real world, we limit the contribution and the tax rates by $0.4-0.4$, respectively. Starting with the optimal fertility, the first line of Table 4 (no pension) shows a marked rise in fertility in parallel with the tax rate from 0.714 (at $\theta=0$ ) to 1.571 (at $\theta=0.4$ ). The first column (not considering the column of contribution rates) of Table 4 (no child allowance) displays a marked sink in fertility while the contribution rate rises: from 0.714 (at $\tau=0$ ) to 0.490 (at $\tau=0.4$ ). Similar tendencies can be observed in the other lines and columns, respectively, though for low enough tax rates (with positive savings), the fertility is paradoxically an increasing function of the contribution rate.

Table 3. Transfer rates and fertility $(n)$

\begin{tabular}{lccccc}
\hline \multicolumn{5}{c}{ T a x r a t e $(\theta)$} \\
\multicolumn{1}{c}{0.0} & 0.1 & 0.2 & 0.3 & 0.4 \\
Contribution & & & & & \\
rate $(\tau)$ & & & & & \\
\hline 0.0 & 0.714 & 0.929 & 1.143 & 1.357 & 1.571 \\
0.1 & 0.680 & 0.907 & 1.134 & 1.347 & 1.551 \\
0.2 & 0.642 & 0.857 & 1.061 & 1.265 & 1.469 \\
0.3 & 0.571 & 0.776 & 0.980 & 1.184 & 1.388 \\
0.4 & 0.490 & 0.694 & 0.898 & 1.102 & 1.306 \\
\hline Remark. $p=0.35, R=1.3$ and $\gamma=0.4$ &
\end{tabular}

Next we present the dependence of the relative efficiency of the transfer system on the transfer rates, in terms of the no-transfer case. The first line of Table 3 (no pension system) displays the drop in the relative welfare as the tax rate rises: from 1 (at $\theta=0$ ) to 0.702 (at $\theta=0.4$ ). The first column of Table 4 (no child allowance) displays the drop in the relative welfare as the contribution rate rises: from 1 (at $\tau=0$ ) to 0.843 (at $\tau=0.4$ ). No other column and only the next row, however, show similar monotonicity. The fourth line and the 
fourth column contains the entry of the approximately maximal efficiency, namely 1.106 at $(\tau, \theta)=(0.3,0.3)$. In this sense, the social optimum is achieved for the careful harmonization of the contribution and the tax rates.

Table 4. Transfer rates and relative efficiency

\begin{tabular}{lccccc}
\hline \multicolumn{7}{c}{ T ax r a te $(\theta)$} \\
& 0.0 & 0.1 & 0.2 & 0.3 & 0.4 \\
Contribution & & & & & \\
rate $(\tau)$ & & & & & \\
\hline 0.0 & 1.000 & 0.948 & 0.879 & 0.796 & 0.702 \\
0.1 & 0.943 & 0.912 & 0.863 & 0.856 & 0.862 \\
0.2 & 0.880 & 0.953 & 1.024 & 1.058 & 1.050 \\
0.3 & 0.879 & 1.007 & 1.083 & 1.106 & 1.070 \\
0.4 & 0.843 & 0.982 & 1.052 & 1.052 & 0.967 \\
\hline
\end{tabular}

To get a more detailed picture, in Table 5 we repeat the calculations made in Tables 3 and 4 along the diagonal with $\tau=\theta$ but display not only the fertility and the relative efficiency rather, also the young- and the old-age consumption and the subjective utility. First of all, note that the optimal saving is only positive for low enough contribution and tax rates, namely below $\tau=\theta=0.15$. The maximum of the subjective utility function is decreasing for all rates. The relative efficiency first decreases, then increases, and finally decreases: its maximum is reached at $\tau_{T}^{*}=\theta_{T}^{*}=0.294$. Note that the introduction of the socially optimal transfer system involves a jump over the gap created by the initial drop of welfare.

\section{Heterogeneous Fertility}

Having discussed the simplest case of homogeneous fertility, we turn to the more realistic and more interesting case of heterogeneous fertility. We shall study the impact of fertilitydependent pensions, first without incentive effects, then with incentive effects.

\subsection{Heterogeneous fertility without incentives (models 2 and 3)}

To derive heterogeneity in the optimally chosen number of children, we shall assume the existence of two types indexed as $i=1,2$. They differ in the following parameter values: the cost of rearing a child $p_{i}$ and the relative utility of having a child $\gamma_{i}$, with the following plausible orderings (cf. CGP):

$$
p_{1}>p_{2}>0 \text { and } 0<\gamma_{1}<\gamma_{2} \text {. }
$$

In words: type 1 spends more money on raising a child than type 2 does and enjoys it less. The corresponding population
Table 5. The impact of the equal transfer rates

\begin{tabular}{|c|c|c|c|c|c|c|}
\hline \multicolumn{7}{|l|}{ Equal } \\
\hline \multicolumn{3}{|l|}{ transfer } & Young-age & \multicolumn{2}{|c|}{ Old-age LT utility } & \multirow[t]{2}{*}{ Relative } \\
\hline rates & Fertility & Saving & onsumption & function & efficiency & \\
\hline$\tau=\theta$ & $n^{*}$ & $s^{*}$ & $c^{*}$ & $e^{*}$ & $U^{*}$ & $\varepsilon$ \\
\hline 0.00 & 0.714 & 0.125 & 0.625 & 0.163 & -0.968 & 1.000 \\
\hline 0.05 & 0.808 & 0.085 & 0.582 & 0.151 & -1.004 & 0.954 \\
\hline 0.10 & 0.907 & 0.039 & 0.544 & 0.141 & -1.040 & 0.912 \\
\hline 0.15 & 1.000 & 0.000 & 0.500 & 0.150 & -1.073 & 0.919 \\
\hline 0.20 & 1.061 & 0.000 & 0.429 & 0.212 & -1.134 & 1.024 \\
\hline 0.25 & 1.122 & 0.000 & 0.357 & 0.281 & -1.238 & 1.087 \\
\hline 0.30 & 1.184 & 0.000 & 0.286 & 0.355 & -1.392 & 1.106 \\
\hline 0.35 & 1.245 & 0.000 & 0.214 & 0.436 & -1.619 & 1.071 \\
\hline 0.40 & 1.306 & 0.000 & 0.143 & 0.522 & -1.969 & 0.967 \\
\hline
\end{tabular}

Remark. LT=Lifetime

shares are denoted by $f_{1}, f_{2}>0$, their sum being equal to 1 : $f_{1}+f_{2}=1$. Then the average number of children is given by

$$
n=f_{1} n_{1}+f_{2} n_{2} .
$$

The aggregate child allowance rule simply remains $\theta=\varphi n$ to be paid by both types but type $i$ receives allowance $\varphi n_{i}$.

Note that here we shall assume that type $i$ 's pension is partly dependent on the type-specific fertility $n_{i}$, but for the time being, the types do not take this into account. Let $\delta \in[0,1]$ be the weight of the common and $\hat{\delta}=1-\delta$ be the weight of the individual pension:

$$
\eta_{i}=\delta \tau n+\hat{\delta} \tau n_{i}, \quad i=1,2 .
$$

If $\delta=0$, then we have two separate pension systems for the low and the high efficiency types. If $\delta=1$, then we have a unified pension system: model 2 . In general, we shall assume $0<\delta<1$ but eventually we shall study the two extreme cases.

Young-age and old-age consumptions are respectively

$$
c_{i}=\hat{\tau}-\theta-\left(p_{i}-\varphi\right) n_{i}-s_{i} \text { and } e_{i}=R s_{i}+\eta_{i} .
$$

The lifetime utility functions are as follows:

$$
U_{i}\left(c_{i}, n_{i}, e_{i}\right)=\log c_{i}+\gamma_{i} \log n_{i}+\beta \log e_{i} .
$$

Substituting (11) into (12) yields the type-specific reduced utility functions:

$$
\begin{gathered}
u_{i}\left(s_{i}, n_{i}\right)=\log \left(\hat{\tau}-\theta-\left(p_{i}-\varphi\right) n_{i}-s_{i}\right)+\gamma_{i} \log n_{i} \\
+\beta \log \left(R s_{i}+\eta_{i}\right) .
\end{gathered}
$$

We solve the individual optimization problems for slack and tight credit constraints separately-without taking into account (10). 
In the case of slack credit constraint, taking the partial derivatives with respect to $s_{i}$ and $n_{i}$ (cf. (4)), yields

$$
c_{i}^{*}=\frac{\hat{\tau}-\theta+R^{-1} \eta_{i}}{1+\beta+\gamma_{i}} \text { and } n_{i}^{*}=\frac{\gamma_{i} c_{i}^{*}}{p_{i}-\varphi} .
$$

In the case of tight credit constraint, we return to (7):

$$
\left(p_{i}-\varphi\right) n_{i}=\gamma_{i} c_{i}=\gamma_{i}\left[\hat{\tau}-\theta-\left(p_{i}-\varphi\right) n_{i}\right] .
$$

With rearrangement, and using again notation $\bar{\gamma}_{i}=1+\gamma_{i}$,

$$
\bar{\gamma}_{i}\left(p_{i}-\varphi\right) n_{i}=\gamma_{i}(\hat{\tau}-\theta) .
$$

Note that it is possible that one type has a slack credit constraint and the other has a tight one. There are four possible combinations:

$$
s_{1}, s_{2}>0, \quad s_{1}>0=s_{2}, \quad s_{2}>0=s_{1}, \quad s_{1}=0=s_{2} .
$$

To avoid unsolvable complexities, we confine our attention to the most important and the simplest case, when both types save nothing. Then we have the following trivial observation (cf. (16)).

Lemma 2. If both types have tight credit constraints: $s_{1}=$ $s_{2}=0$, then type 1 is less fertile than type 2 :

$$
p_{1}>p_{2}>0 \text { and } 0<\gamma_{1}<\gamma_{2} \text { implies } n_{1}^{*}<n_{2}^{*} \text {. }
$$

To determine the individual optimum, we must calculate the equilibrium value of $\varphi$.

Theorem 5. Consider a fertility-specific transfer system, where the workers do not take into account this feature. In the tight-tight optimum, the per-child allowance $\varphi$ is the smaller positive root of the following quadratic equation:

$$
A \varphi^{2}-B \varphi+C=0,
$$

where the coefficients in (18) are respectively

$$
\begin{gathered}
A=\omega \bar{\gamma}_{1} \bar{\gamma}_{2}+f_{1} \gamma_{1} \bar{\gamma}_{2}+f_{2} \gamma_{2} \bar{\gamma}_{1}, \\
B=\omega \bar{\gamma}_{1} \bar{\gamma}_{2}\left(p_{1}+p_{2}\right)+f_{1} \gamma_{1} \bar{\gamma}_{2} p_{2}+f_{2} \gamma_{2} \bar{\gamma}_{1} p_{1}, \\
C=\bar{\gamma}_{1} \bar{\gamma}_{2} p_{1} p_{2},
\end{gathered}
$$

and

$$
\omega=\frac{\theta}{\hat{\tau}-\theta} \text { and } \bar{\gamma}_{i}=1+\gamma_{i}, \quad i=1,2 .
$$

Finally, for transfer pair $t=(\tau, \theta)$, we define the paternalistic utilitarian social welfare function:

$$
\begin{aligned}
V(t, \delta) & =\sum_{i=1}^{2} f_{i} V_{i}\left[t, \delta, n_{i}^{*}\right], \text { where } V_{i}\left[t, \delta, n_{i}^{*}\right] \\
& =\log c_{i}^{*}+\gamma \log n_{i}^{*}+\log e_{i}^{*} .
\end{aligned}
$$

We start with a trivial consequence of the Jenseninequality and (10b).

Theorem 6. The stronger the differentiation of the pensions, i.e. the lower the $\delta$, the lower is the social welfare. In particular, for any transfer pair $t, V(t, 0)<V(t, 1)$.
Example 2. (Model 2.) We assume that the shares of the two types are equal: $f_{1}=f_{2}=0.5$; and differentiate the costs and utilities of child between the two types: $p_{1}=0.3$, $p_{2}=0.4$ and $\gamma_{1}=0.35, \gamma_{2}=0.45$. For the time being, the pension benefits are uniform: $\delta=1$.

Table 6 depicts the behavior of model 2. Regardless of heterogeneity, at the optimum, the two transfer rates are again equal. Raising them (above 0.2-0.2) diminishes type 1's fertility, though still increases the average fertility. The basic

\begin{tabular}{|c|c|c|c|c|c|}
\hline \multirow{2}{*}{$\begin{array}{l}\text { Equal } \\
\text { transfer }\end{array}$} & \multirow[b]{3}{*}{ Fertility-1 } & \multirow[b]{3}{*}{ Fertility-2 } & \multicolumn{2}{|c|}{ Paternalistic } & \multirow{3}{*}{$\begin{array}{l}\text { Relative } \\
\text { efficiency }\end{array}$} \\
\hline & & & Utility-1 & Utility-2 & \\
\hline rates & & & \multicolumn{2}{|c|}{ function } & \\
\hline$\tau=\theta$ & $n_{1}^{*}$ & $n_{2}^{*}$ & $V_{1}^{*}$ & $V_{2}^{*}$ & $\varepsilon$ \\
\hline 0.20 & 0.703 & 1.535 & -2.431 & -2.186 & 0.923 \\
\hline 0.22 & 0.696 & 1.602 & -2.382 & -2.115 & 0.951 \\
\hline 0.24 & 0.686 & 1.672 & -2.348 & -2.056 & 0.973 \\
\hline 0.26 & 0.672 & 1.749 & -2.329 & -2.010 & 0.989 \\
\hline 0.28 & 0.653 & 1.831 & -2.326 & -1.977 & 0.998 \\
\hline 0.30 & 0.630 & 1.920 & -2.339 & -1.956 & 1.000 \\
\hline 0.32 & 0.601 & 2.016 & -2.371 & -1.948 & 0.994 \\
\hline 0.34 & 0.565 & 2.121 & -2.423 & -1.957 & 0.979 \\
\hline 0.36 & 0.523 & 2.235 & -2.501 & -1.983 & 0.954 \\
\hline
\end{tabular}
reason for this paradoxical behavior is probably connected to the introduction of net transfers from type 1 to type 2 via the child allowances. While the socially optimal contribution rate remains close to 0.3 , the type-specific optimum arises earlier (at $\tau_{1}=0.28$ ) for type 1 and later (at $\tau_{2}=0.32$ ) for type 2. Since we do not deal with slack constraints, the value of the social welfare function at no transfer is unknown. Therefore we must take the maximum as our starting point for measuring the relative efficiency of the system.

Table 6. The impact of equal transfer rates: heterogeneous types, uniform pensions

Remark. $p_{1}=0.4, p_{2}=0.3$ and $\gamma_{1}=0.35, \gamma_{2}=0.45$.

Next we turn to the differentiated pensions: $0 \leq \delta<1$. For the time being, we exclude incentive effects. To obtain a marked difference, we consider the maximal differentiation with $\delta=0$. The outcomes displayed in Table 7 are basically the same as before: the fertilities remain invariant, but the less fertile type receives even less utility, the more fertile receives even higher utility and in balance, the maximal social welfare is more than 6 percent less than with uniform pensions and reached at transfer rates close to 0.26 rather than to 0.3 . Note that the type-specific optima are achieved now for much lower (at $\theta_{1}=\tau_{1}=0.22$ ) and much higher transfer rates (at $\theta_{2}=\tau_{2}=0.34$ ), signaling polarization.

What happens if the types $d o$ take into account the fertilitydependence of the child allowance and the pension? 
Table 7. The impact of transfer rates: types, totally differentiated pensions without incentives

\begin{tabular}{lccccc}
\hline Equal & & \multicolumn{5}{c}{ P a t e r n l i s t c c } \\
transfer & & & Utility-1 & Utility-2 & Relative \\
rates & Fertility-1 & Fertility-2 & f u n c t i o n & efficiency \\
$\tau=\theta$ & $n_{1}^{*}$ & $n_{2}^{*}$ & $V_{1}^{*}$ & $V_{2}^{*}$ & $\varepsilon$ \\
\hline 0.20 & 0.703 & 1.535 & -2.896 & -1.870 & 0.889 \\
0.22 & 0.696 & 1.602 & -2.883 & -1.783 & 0.912 \\
0.24 & 0.686 & 1.672 & -2.890 & -1.707 & 0.928 \\
0.26 & 0.672 & 1.749 & -2.918 & -1.642 & 0.936 \\
0.28 & 0.653 & 1.831 & -2.969 & -1.589 & 0.937 \\
0.30 & 0.630 & 1.920 & -3.045 & -1.546 & 0.929 \\
0.32 & 0.601 & 2.016 & -3.150 & -1.516 & 0.912 \\
0.34 & 0.565 & 2.121 & -3.289 & -1.500 & 0.884 \\
0.36 & 0.523 & 2.235 & -3.471 & -1.500 & 0.845 \\
\hline
\end{tabular}

Remark. See Table 6.

\subsection{Fertility-dependent pensions with incentives (model 4)}

Introducing the game-theoretic notation $-i$ as the 'other' player's index, similarly to (3b), (3) becomes

$$
\begin{gathered}
u_{i}\left(0, n_{i}, n_{-i}\right)=\log c_{i}\left(0, n_{i}, n_{-i}\right)+\gamma_{i} \log n_{i} \\
+\beta \log e_{i}\left(0, n_{i}, n_{-i}\right), \quad i=1,2 .
\end{gathered}
$$

Taking the partial derivatives, (7) is modified into

$u_{i, n_{i}}^{\prime}\left(0, n_{i}, n_{-i}\right)=\frac{-\left(p_{i}-\varphi\right)}{\hat{\tau}-\theta-\left(p_{i}-\varphi\right) n_{i}}+\frac{\gamma_{i}}{n_{i}}+\frac{\hat{\delta} \beta}{\delta n+\hat{\delta} n_{i}}=0$,

$$
i=1,2 \text {. }
$$

If $0<\delta<1$, then we have a system of two nonlinear equations, which is probably only solvable with numerical methods. Moreover, we do not want to consider a Cournotgame between less and more fertile types.

However, for total separation of pensions: $\delta=0$, i.e. $\hat{\delta}=$ $1,(22 *)$ breaks up into two independent equations:

$$
\frac{-\left(p_{i}-\varphi\right)}{\hat{\tau}-\theta-\left(p_{i}-\varphi\right) n_{i}}+\frac{\gamma_{i}}{n_{i}}+\frac{\beta}{n_{i}}=0, \quad i=1,2 .
$$

Comparing (22) and (22), the only difference between them is that $\gamma_{i}$ in (22) is replaced by $\gamma_{i}+\beta$ in $(\tilde{22)}$. This observation proves

Theorem 7. a) For total separation of pensions $(\delta=0)$, introducing the incentive effects is equivalent to adding the discount factor $\beta$ to the relative child utility coefficient $\gamma_{i}$ in model 3:

$$
\tilde{\gamma}_{i}=\gamma_{i}+\beta .
$$

b) The activation of incentives obviously raises the total fertility but decreases the lower type's fertility.
The relations become so complicated that the analytical investigation of the social welfare must be replaced by numerical trials.

We repeat the numerical calculations with the new $\tilde{\gamma}_{i}$ s. Table 8 shows that type 2's fertility increases, the socially optimal contribution and tax rates get lower, around 0.26 , the scissors between the two types' utilities open up and the social welfare is higher than without incentives and with dif-

\begin{tabular}{|c|c|c|c|c|c|}
\hline \multirow{2}{*}{$\begin{array}{l}\text { Equal } \\
\text { transfer }\end{array}$} & \multirow[b]{3}{*}{ Fertility-1 } & \multicolumn{4}{|c|}{ Paternalistic } \\
\hline & & & Utility-1 & Utility 2 & Relative \\
\hline rates & & Fertility-2 & & nction & efficiency \\
\hline$\tau=\theta$ & $n_{1}^{*}$ & $n_{2}^{*}$ & $V_{1}^{*}$ & $V_{2}^{*}$ & $\varepsilon$ \\
\hline 0.20 & 0.878 & 1.660 & -2.760 & -1.785 & 0.940 \\
\hline 0.22 & 0.867 & 1.708 & -2.753 & -1.711 & 0.959 \\
\hline 0.24 & 0.853 & 1.761 & -2.766 & -1.648 & 0.971 \\
\hline 0.26 & 0.835 & 1.818 & -2.799 & -1.595 & 0.976 \\
\hline 0.28 & 0.813 & 1.881 & -2.854 & -1.552 & 0.973 \\
\hline 0.30 & 0.785 & 1.951 & -2.933 & -1.518 & 0.962 \\
\hline 0.32 & 0.752 & 2.029 & -3.041 & -1.494 & 0.942 \\
\hline 0.34 & 0.712 & 2.117 & -3.184 & -1.482 & 0.912 \\
\hline 0,36 & 0,663 & 2,215 & $-3,369$ & $-1,484$ & 0,870 \\
\hline
\end{tabular}
ferentiation (4 percent) but lower than without incentives and differentiation ( 2 percent).

Table 8. The impact of transfer rates: heterogeneous types, totally differentiated pensions with incentives

Remark. See Table 6.

\section{Conclusions}

We have revisited GLM's Siamese twins of child support and public pension, at least in its equilibrium framework. In our models, public pension is important because the young are short-sighted and the large families had been broken up. Child support is also important because the introduction of public pension may undermine fertility. We have emphasized the importance of modeling credit constraints and have distinguished the slack and the tight credit constraints in modifying GLM's model.

A surprising feature of this type of models is as follows: starting from the no-transfer system, the social welfare decreases as the mandatory pension contribution crowds out the more efficient private saving. Only after surpassing the slack-tight boundary (the separatrix) will the social welfare increase. To increase welfare with respect to no-transfer, the government transfers must jump the gap created by initially declining welfare!

An important but largely neglected dimension of the fertility issue is heterogeneity: there are families with low fertil- 
ity and others with high fertility produced by high-cost, lowutility and low-cost, high-utility types, respectively. Here the redistribution between the two types in the child allowances cannot be forgotten. In various countries (including Germany and Hungary) there are also plans to make the pension benefits to be partly proportional to fertility. We are able to model such a design and at least on paper, we can prove that such a transfer system increases the average fertility. Nevertheless, there are strong reasons against its application. To name just two: such a policy probably diminishes the social welfare and-leaving the realm of our model-may polarize the society into fertile and infertile.

\section{Appendix}

\section{A Proof of Theorem 1b}

Take the derivative of $n_{S}^{*}(\tau, \theta)[(6)]$ with respect to $\tau$ and drop the constants:

$\frac{\partial n_{S}^{*}(\tau, \theta)}{\partial \tau} \approx \gamma\left[(1+\beta+\gamma) p-\gamma R^{-1} \tau\right]+[\gamma \hat{\tau}+\theta(1+\beta)] \gamma R^{-1}$.

A simple calculation yields that

$$
\frac{\partial n_{S}^{*}(\tau, \theta)}{\partial \tau}<0 \text { if and only if } \theta<\theta^{\circ} .
$$

\section{B Proof of Theorem 2}

In this proof, we shall drop the subindex $\mathrm{S}$ and study the behavior of the social welfare function

$$
V(\tau, \theta)=\log c(\tau, \theta)+\gamma \log n(\tau, \theta)+\log e(\tau, \theta) .
$$

Take the partial derivatives with respect to the two transfer rates:

$$
V_{\tau}^{\prime}(\tau, \theta)=\frac{c_{\tau}^{\prime}(\tau, \theta)}{c(\tau, \theta)}+\gamma \frac{n_{\tau}^{\prime}(\tau, \theta)}{n(\tau, \theta)}+\frac{e_{\tau}^{\prime}(\tau, \theta)}{e(\tau, \theta)}
$$

and

$$
V_{\theta}^{\prime}(\tau, \theta)=\frac{c_{\theta}^{\prime}(\tau, \theta)}{c(\tau, \theta)}+\gamma \frac{n_{\theta}^{\prime}(\tau, \theta)}{n(\tau, \theta)}+\frac{e_{\theta}^{\prime}(\tau, \theta)}{e(\tau, \theta)} .
$$

Using $e=\beta R c$, the first and the third terms are equal in both equations. Moreover, using (6) at $\tau=0=\theta$

$$
\begin{gathered}
\frac{c_{\tau}^{\prime}(0,0)}{c(0,0)}=\frac{\gamma p^{-1} R^{-1}-(\bar{\gamma}+\beta)}{\bar{\gamma}+\beta}<0 \\
\text { and } \frac{n_{\tau}^{\prime}(0,0)}{n(0,0)}=\gamma^{-1} \frac{c_{\tau}^{\prime}(0,0)}{c(0,0)} .
\end{gathered}
$$

Hence, by the linearity of $c(\tau, 0)$ and $n(\tau, 0)$, the elasticities are equal to 1 , i.e.

$$
V_{\tau}^{\prime}(0,0)=3 \frac{c_{\tau}^{\prime}(0,0)}{c(0,0)}<0
$$

Similarly for $\theta$, the elasticities are 1 and -1 , i.e. $V_{\theta}^{\prime}(0,0)=$ $-2+\gamma<0$ etc.

The second-order conditions are omitted.

\section{Proof of Lemma 1}

Inserting respectively the formulas (6c) and (8a) for $n_{S}^{*}$ and $n_{T}^{*}$ into the separatrix equation $n_{S}^{*}=n_{T}^{*}$ yields

$$
\frac{\gamma \hat{\tau}+(1+\beta) \theta}{(1+\beta+\gamma) p-\gamma R^{-1} \tau}=\frac{\gamma \hat{\tau}+\theta}{\bar{\gamma} p} .
$$

With rearrangement, $[\gamma \hat{\tau}+(1+\beta) \theta] \bar{\gamma} p=[\gamma \hat{\tau}+\theta][(1+\beta+$ $\left.\gamma) p-\gamma R^{-1} \tau\right]$. After simplification, $\theta(\tau)$ is obtained. For $\theta>\theta(\tau), s(\tau, \theta)=0$; for $\theta<\theta(\tau), s(\tau, \theta)>0$.

The separatrix is a fraction: its numerator is the product of two decreasing positive functions and its denominator is an increasing positive function, therefore the fraction is also declining.

\section{Proof of Theorem 4}

At the tight credit constraint, our starting point is as follows:

$V\{\tau, \theta\}=\log \frac{\hat{\tau}-\theta}{\bar{\gamma}}+\gamma \log \frac{\gamma \hat{\tau}+\theta}{\bar{\gamma} p}+\log \frac{\tau(\gamma \hat{\tau}+\theta)}{\bar{\gamma} p} \rightarrow \max$.

Using the identity $\log (x / y)=\log x-\log y$, the constant denominators can be dropped. Reintroducing $\hat{\tau}=1-\tau$, we have then an equivalent problem:

$W\{\tau, \theta\}=\log (1-\tau-\theta)+\bar{\gamma} \log (\gamma(1-\tau)+\theta)+\log \tau \rightarrow \max$.

Taking the partial derivatives of $W$ with respect to $\tau$ and $\theta$ and equate the derivatives to zero yield the first-order necessary conditions:

$$
0=W_{\tau}^{\prime}=\frac{-1}{1-\tau-\theta}-\frac{\bar{\gamma} \gamma}{\gamma(1-\tau)+\theta}+\frac{1}{\tau}
$$

and

$$
0=W_{\theta}^{\prime}=\frac{-1}{1-\tau-\theta}+\frac{\bar{\gamma}}{\gamma(1-\tau)+\theta} .
$$

From (A2),

$$
\theta=\frac{1-\tau}{2+\gamma}=\kappa(1-\tau) .
$$

Inserting back (A3) into (A1),

$$
\frac{-1}{(1-\kappa)(1-\tau)}-\frac{\bar{\gamma} \gamma}{(\gamma+\kappa)(1-\tau)}+\frac{1}{\tau}=0
$$

Hence Theorem 4 is obtained.

\section{E Proof of Theorem 5}

Using $\bar{\gamma}_{i}=1+\gamma_{i}$ in (16) and inserting the new formula

$$
n_{i}=\frac{\gamma_{i}(\hat{\tau}-\theta)}{\bar{\gamma}_{i}\left(p_{i}-\varphi\right)}
$$

into $\theta=\varphi\left(f_{1} n_{1}+f_{2} n_{2}\right)$ yields an implicit equation for $\varphi$ :

$$
\theta=\varphi\left(f_{1} \frac{\gamma_{1}(\hat{\tau}-\theta)}{\bar{\gamma}_{1}\left(p_{1}-\varphi\right)}+f_{2} \frac{\gamma_{2}(\hat{\tau}-\theta)}{\bar{\gamma}_{2}\left(p_{2}-\varphi\right)}\right) .
$$

Having eliminated the denominators yields the quadratic equation (18)-(20) for $\varphi$. It is the smaller root, which satisfies $0<\varphi<p_{2}<p_{1}$, etc. 


\section{Acknowledgements}

I express my indebtedness to Róbert Iván Gál for his permanent encouragement to pursue this topic, to Hans Fehr, Volker Meier, János Vincze and anonymous referees for their thorough critique. The author has received generous financial support from OTKA K 81483.

\section{REFERENCES}

[1] Barr, N. and Diamond, P. (2008): Reforming Pensions: Principles and Policy Choices, Oxford, Oxford University Press.

[2] Becker, G. S. (1960): “An Economic Analysis of Fertility”, Demographic and Economic Change in Developed Countries, eds: R. Easterline, Princeton, Princeton University Press.

[3] Boldrin, M; De Nardi, M. and Jones, L. E. (2005): "Fertility and Social Security", NBER Working Paper 11146.

[4] Cigno, A. (1992): "Children and Pension", Journal of Population Economics 5, 175-183.

[5] Cremer, H., Gahvari, F. and Pestieau, P. (2006): "Pensions with Endogenous and Stochastic Fertility”, Journal of Public Economics 90, 2303-2321.

[6] Cremer, H., Gahvari, F. and Pestieau, P. (2008): "Pensions with Heterogeneous Agents and Endogenous Fertility", Journal of Population Economics 21, 961-981.

[7] Eckstein, Z. and Wolpin, K. I. (1985): "Endogenous Fertility and Optimal Population Size", Journal of Public Economics 75, 93-106.

[8] Feldstein, M. S. (1985): "The Optimal Level of Social Benefits", Quarterly Journal of Economics 100, 302-320.

[9] Fenge, R. and Meier, R. (2005): "Pension and Fertility Incentives", Canadian Journal of Economics 38, 28-48.
[10] Fenge, R. and Meier, R. (2009): "Are Family Allowances and Fertility Related Pensions Perfect Substitutes?", International Taxation and Public Finance 16, 137-163.

[11] Galasso, V.; Gatti, R. and Profeta, P. (2009): "Investing for the Old Age: Pensions, Children and Savings", International Taxation and Public Finance 16, 538-559.

[12] van Groezen, B.; Leers, Th. and Meijdam, L. (2003): "Social Security and Endogenous Fertility: Pensions and Child Allowances as Siamese Twins", Journal of Public Economics 87, 233-251.

[13] van Groezen, B. and Meijdam, L. (2008): "Growing Old and Staying Young: Population Policy in an Aging Closed Economy", Journal of Population Economics 21, 573-588.

[14] Leibenstein, H. M. (1957): Economic Backwardness and Economic Growth, New York, Wiley.

[15] Lindbeck, A., Nyberg, S. and Weibull, J. W. (1999): "Social Norms and the Welfare State", Quarterly Journal of Economics 114, 1-35.

[16] Rangel, A. (2003): Forward and Backward Intergenerational Goods: Why Is Social Security Good for the Environment?, American Economic Review 93, 813-834.

[17] Simonovits, A. (2013): The Socially Optimal Cap on Pension Contributions, IE-CERS-HAS Working Paper 36.

[18] Simonovits, A. (2014): "Optimal Child Allowances with Heterogeneous Fertilities", IE-CERS-HAS Working Paper 1.

[19] Simonovits, A. (2015): Socially Optimal Child-related Transfers with Endogenous Fertility, IE-CERS-HAS Working Paper 37. 